\title{
Instabilités de fonctionnement à débit partiel d'un inducteur fretté : comparaison avec le cas non fretté
}

\author{
par C. Offtinger, C. Henry, R. Morel \\ Equipe Hydraulique INSA de Lyon \\ Laboratoire de Mécanique des Fluides el d'Acoustique
}

\section{INTRODUCTION}

Ln inducteur, ou roue de gavage, est une rour axiale placée à l'amont d'une pompe afin d'amćliorer se capacité d'aspiration. Cependant, l'inconvénient majeur de ce type de roue est son comportement instable à débit partiei. De nombreux auteurs dont Acosta [21, Janigro el Ferrini [3], Laksiminarayana [4] et Yedidiah [5] ont décrit les principates figures de cavitation responsables des fluctuations de pression et de débit.

L'élude cxpérimentale d'un inducteur constitué de trois aubes, placé en alimentation d'une rouc centrifuge réalisée au Laboratoirc de Mécanique des Fluides de I'INSA de Lyon a mis en évidence un comportentent instable de cclui-ci à débil partiêl [1]. Une étude similaire a été entreprisc sur le même incỏıcteur ceinturé par une frettc soudéc en tête des aubes.

Pour cet inducteur, une campagne de visualisation a permis de repérer qualitativement l'apparition el l'évolution des différentes formes de cavitation : cavitation d'aubage et cavitation de recirculation. Afin de caractériser chacune des figures mises ainsi en evidence, des mesures de fuetuations de pressions parićtales ont été réalisées dans les condinites amont $\mathrm{cl}$ aval ì l'aide de captetrs de pression instaitonnairc. Les résultats de cete étude sonil comparés à ceux obtenus avec l'incuucteur non fretté.

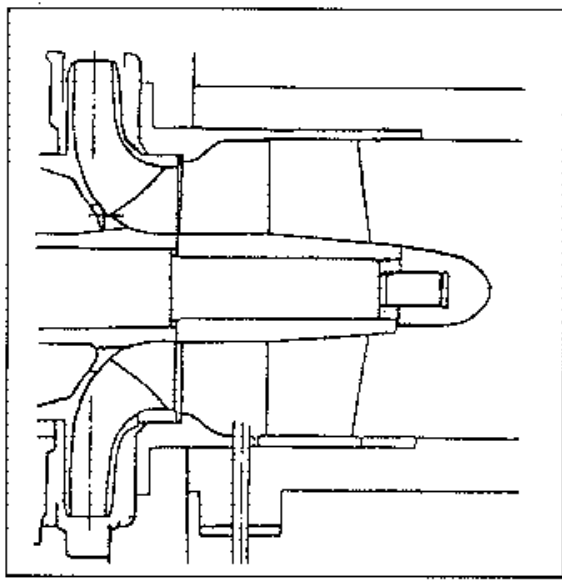

1. Schéma de montage de l'inducteur.

\begin{tabular}{|c|c|}
\hline & NOTATIONS \\
\hline $\mathrm{g}$ & accélération de la pesanteur \\
\hline $\mathrm{r}$ & rayon \\
\hline $\mathrm{Q}$ & débit volumique \\
\hline NPSH & Net Positive Suction Head \\
\hline $\mathrm{U}$ & vitesse périphérique \\
\hline$\sigma$ & NPSH adimensionné $\quad \sigma=g$ SPSH/L: \\
\hline$\delta$ & coefficient de débit $\delta=Q / U, \mathrm{r}^{2}$ \\
\hline indice : & \\
\hline$p$ & picd \\
\hline $\mathrm{t}$ & : tête \\
\hline disp & disponiblc \\
\hline
\end{tabular}

Tableau 1: Caractéristiques de l'inducteur:

\begin{tabular}{|c|c|}
\hline Vitesse de rotation & $2960 \mathrm{tr} / \mathrm{min}$ \\
\hline Nombre d aubes & 3 \\
\hline Coefficient de débil de dessin & 0,14 \\
\hline Vitesse spécifique d'aspiration & 456,45 \\
\hline Rapport de rayon pied / tête en entrée & $r_{r} / r_{1}=0,354$ \\
\hline Rappont de rayon picd / tête en sortie & $r_{\sqrt{2} / r}=0,393$ \\
\hline $\begin{array}{l}\text { Cocfficicnt de débil dc } \\
\text { meilleur rencement }\end{array}$ & 0,113 \\
\hline Jeu radial & $0,0011 r_{i}^{\grave{a}} 0,0044 r$ \\
\hline
\end{tabular}

\begin{tabular}{|l|l|l|}
\hline & Tête & Pied \\
\hline Epaisseur relative des aubes & 0,0118 & 0.0412 \\
\hline Solidité & 1,6 & 1,77 \\
\hline Angle de calage & $80,35^{\circ}$ & $61,51^{\circ}$ \\
\hline Angle géométrique en entrée & $82,15^{\circ}$ & $67,7^{\circ}$ \\
\hline Angle géométrique en sortic & $78,55^{\circ}$ & $55,32^{\circ}$ \\
\hline
\end{tabular}




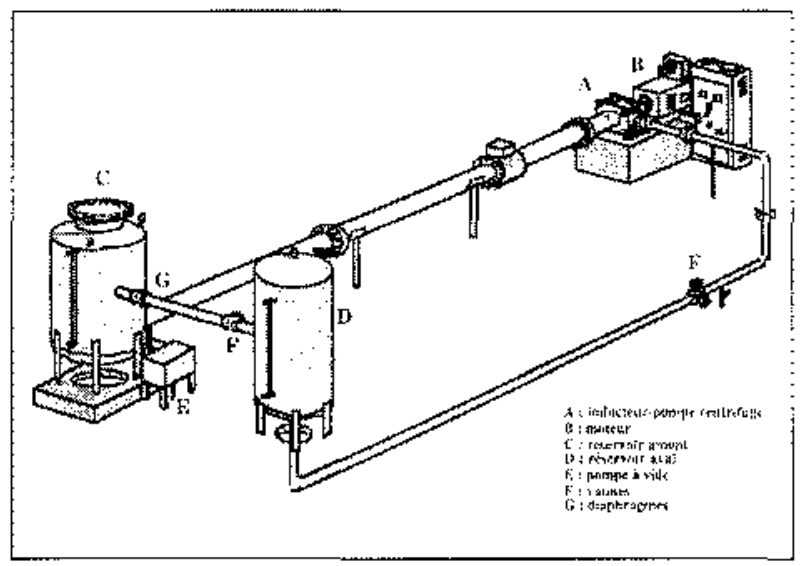

2. Boucle d'essais.

\section{INDUCTELR ET BOUCLE D'ESSAIS}

L'inducteur testé est placé en amont d'une pompe centrilige sur le même arbre que celle-ci. Les principaics caractéristiques de l'inducteir sont données dans le tablea: $:$.

Le monlage de la pompe centrifuge ct de l'inducteur dans les deux configurations fretté et non fretić est schématisć figure 1.

Les essais ont été menés en cau dans un circuit en boucle ícrméc comme le montre la figurc 2 . Ce circuit csi composé principalement du groupe pompe centrifuge - incucteur (A) entrâेné par un moteur à courant continu (B), de deux réservoirs (C) $\mathrm{cl}$ (D) de capacité respective $2.6 \mathrm{~m}$ ' $12.2 \mathrm{~m}^{3}$, d'une pompe à vide à anneau d'eau (E), de vannes de rćglage de débil (F) el de diaphragmes interchangeables (G).

Dans le réservoir d'aspiration (C) la modification de la hauteur d'eau ou l'ajustement du niveau de pression au dessus de la surface libre ats moyen de la pompe à vide permet de faire varier la hauleur de charge nette absoluc sisponible $\mathrm{NPSH}_{\text {cisp }}$ à l'entréc de l'incucteur.

Une manchette cn altuglass remplaçant le carter à l'aspiration de la roue centrifige permei de visualiser "'écoulement en amont ct à travers l'inducteur ê de pholographicr ou lilmer les Gifférentes formes dc cavitaison.

Les débits à l'amont et à l'aval de la machine sont mesurés à l'aice de débitmètres électromagnétiques Endress Hauser. Le débit maximum cans la boucle pout une vilesse de rotation ce $3000 \mathrm{tr} / \mathrm{min}$ est de $0, \mathrm{l} \mathrm{m}^{3 / \mathrm{s}}$. L'incertitude sur la mesure du débit varic avec la vitesse de l'écoulement : pour une viicsse comprise ente $1 \mathrm{cl} 10 \mathrm{~m} / \mathrm{s}, \pm 1 \%$ de 20 à $100 \%$ de l'ćchelle de mests$\mathrm{re}, \pm 2 \%$ de 0 à $20 \%$ de l'ćchelle de mesure. L'incertitude est doublée pour une vilesse comprise entre $0,5 \mathrm{ct} 1 \mathrm{~m} / \mathrm{s}$.

Un couplemètre dynamique Staiger Mohito, de fréquence d'échantilionage $600 \mathrm{~Hz}$, placé sur l'arbre cntre le moleur et la pompe, dorne accès au couple ei à la vitesse de rotation. L'étenčue de mesure est $500 \mathrm{Nm}$ avec une précision (linéarité ct hystéresis) de $\pm 0,1 \%$ de l'échelle cntic̀re.

La pression à 1'espiration de la rouc est mesuréc à l'aide d'un capteur de pression absoluc Roscmount 1151 AP dont la gamme de mesure est $0-1,87$ l0 $0^{5}$ á, sa précision est $0, i$ à $0,2 \%$ de l'étendue de mesure utilisée (en l'occurence 0-1,5 10\% $\mathrm{Pa}$ ).

Les mesures de fluctuations de pressions óans les conduites amont et aval sont réalisćes à l'aide de capteurs de pression instationaire de type ENTRAN à membrane piezorésisive. La bande passante de ces capteurs est $13 \mathrm{kH} \%$.

Dans la conduite d'aspiration on utilise trois capteurs dont l'ćtenduc de mesure est l bar, l'erreur de mesure $\pm 1 \%$ de I'étendue đe mesure et la dérive du zćro avec la température $=3250 \mathrm{~Pa} / 50^{\circ} \mathrm{C}$.

Un quatrième capteur, uilisé dans la conduite de refoulement a pour étendue de mesure 7 bar, erreur cic mestre $\pm 0,7 \%$ de l'étendue de mesture et dérive du zéro avec la température $\pm 0,65610^{\circ} \mathrm{Pe} / 50^{\circ} \mathrm{C}$

Les signaux sont enregistrés sur micro ordinateur $\mathrm{PC} 386$ aे l'aide d'une caric d'acquisition 8 voies de type Eurosmart associée à un logiciel de dépouillement de résultats Physcope.

La fréquente d'échantillonnage de l'acọvisition des lluctuations est de $340 \mathrm{~Hz}$. L'actuisition a été réaliséc sans fituage. Ceperdant, nous avons opéré sur les mesures brutes un filtrage numérique passe bas de fréquence de coupure $160 \mathrm{~Hz}$ pour nous placer dans les mêmes conditions que pour les mesures sur l'inducteur non rellé

L'ćtalonnage des capteurs instationnaires est réalisé in situ tous les trois points de mesure pour s'affranchir des problèmes liés à la dérive des capteurs en tempéralture. Il faut cependant noter qu'il s'agit d'un étalonnage statique.

\section{III $\square$ VISUALISATIONS}

La campagne de visuaiisations entreprise a permis d'associer une figure de cavitation caractéristique à chaque région du plan $\mathrm{NPSH}_{\text {wip }} / \mathrm{Q}$. Sur la figure 3 sont indiqués les différents domaincs observés, présentés sous forme aưimensionnelle $\sigma_{\text {disp }} / \delta$. A titre de comparaison, la figure 4 rappelle les résultats obtenus par Pagnicr [1] sur I'inductcur non freté.

Une première série d'expériences réalisée en diminuant le débil à NPSH ${ }^{\text {tup }}$ fixé mel en évidence trois régions

- Une région I correspondant à un régime de fonctionnement assez stable ci peu bruyant, où cst observée sniquement une cavitation d'aubage. Cette zone s'étend sur la même plage de débil pour l'inducteur frente et l'inducteur non fretté $(\delta>0,3)$.

On distingue une évolution différentes de part et d'autre du débit de dessin de l'inducteir, $\hat{b}=0,384$ :

- pour des débits supćrieurs ou égaux au débil de cessin de l'inductcur (incidence nulle ou négative), aucune forme de cavitation n'est déccíable sur le côté cxirados des aubes (seul côté visible à cause de la frette).

- pour des débits inférieurs al débit ce dessin (incidence positive), on observe de la cavitation d'aubagc sur le côté externc des aubes (cxurados).

Le défaut d'incidence entraîne un décollement soit sur le côté interne (incidence tégative), soit sur le côté exierne (incidence positive) des aubes. Dans la zone décoliéc, la pression est íajble et peut donner naissance à de la cavitation lorsque $\sigma_{\text {cis }}$ est diminué. Seton la valeur de $\sigma_{\text {dss }}$, on observe soit une "lame" de vapeur atachée à l'ałbage pour des $\sigma_{\text {das }}$ relativement ćievés, soil des grosses bultes de vapeur qualifices de cavitation à bulles séparées pour des $\sigma_{\text {ikip }}$ plus faibles.

- Une région Il oì se forme, à l'aspiration, un tore de recixculation ct de prérotation au voisinage du carter. Ce régime de fonctionnement est accompagné de plus, par un bruit de crépitement très caractéristiquc.

La cavitation de recirculation apparaîl tout d'abord de mantière desorganiscé puis, lorsque le débit cst diminué, des poches de recirculation coniques se forment et s'organisent en trois petits cônes qui remontent l'ćcoulement. Le tore de recirculation se forme alors sur la base de ces trois cones. 


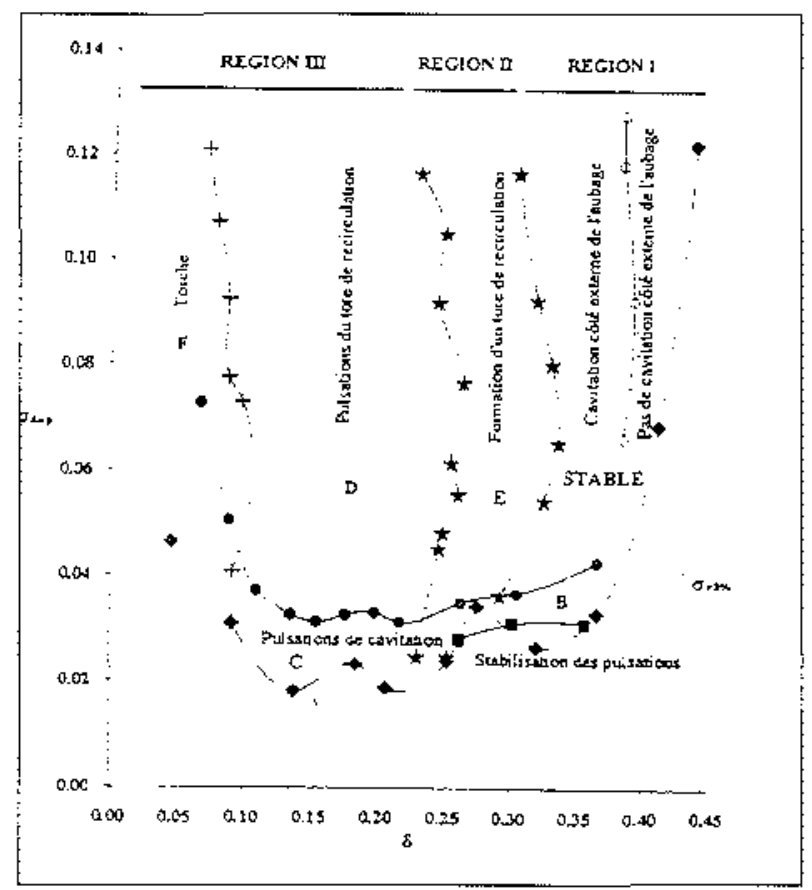

3. Dornaines d'instabilités inducteur fretté.

- Dans une région III le tore de recirculation s'ćtend̀ progressivement vers l'amort at se met à pulser quand le cébje esé diminué. L'écoulement est nettement moirs bruyani ct te bruit caractéristique de la région Il n'est plus percepliblc.

Pour de faibles débits, le tore s'ćtend plus loin à l'amon qu'i] r'est visible au travers de la manchetle transparente $(L=50 \mathrm{~cm})$. Les pulsations du tore apparaissent pour des débils plus élevés $(\delta=0,25$ au ficu ce 0,22$)$ forsque l'inducteur est fretté. Il se peur que la cavitation marginale, présente dars le cas non fretté, désorganise la formation du tore et que l'absence de cetłe figure de cavitation permetic aux autres figtres de s'installer plus facilement .

La zone de tore non pulsé visualisée sans la frette n'a pas pu être mise en évidence.

En diminuant davantage Ic débil, le lore de recirculation se contracte vers l'axe de la conduite d'aspiration et donte naissance à une torche pour $\delta \leq 0,09$ qui s'étend loin vers l'amont. Cette torche se forme alors que le tore de recirculation pulsc encore, elle est instabie et on observe égatement des putsations.

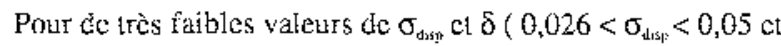
$0,046<\delta<0,114$ ), la torche occupe presque toute la conduite, i1 semble que cclle-ci s'étende foir vers l'amont. Les pulsations de faible fréquence et forle amplitude de ta torche semblent se propager jusọu' au réservoir d'aspiration situé à $9 \mathrm{~m}$ cn amont de l'inducteur.

En eflel, au voisinage du réservoix, on entend dans la conduitc de très violentes implosions qui s'accompagnent de mouvements de la conduite à fabib fréquenec. La torche a un aspect très particulier; cllc est composée de grosses bulles.

Une deuxième séric d'expéricnces réalisée en diminuant NPSHdisp à débit constant, montre que, de manière générale, lorsque NPSHdisp diminue, l'écoulcment devient de plus en plus diphasique el la caviation se mel à pulser puis se stábilise (supercavitation).

Pour un cocfficient de débit $\delta$ supćrieur à 0,275 , cn diminuant le $\sigma_{\text {d:ts, }}$, on observe successivement : une remontée vers l'amont de petits filaments tourbillonnaires cavitants qui se détachent de

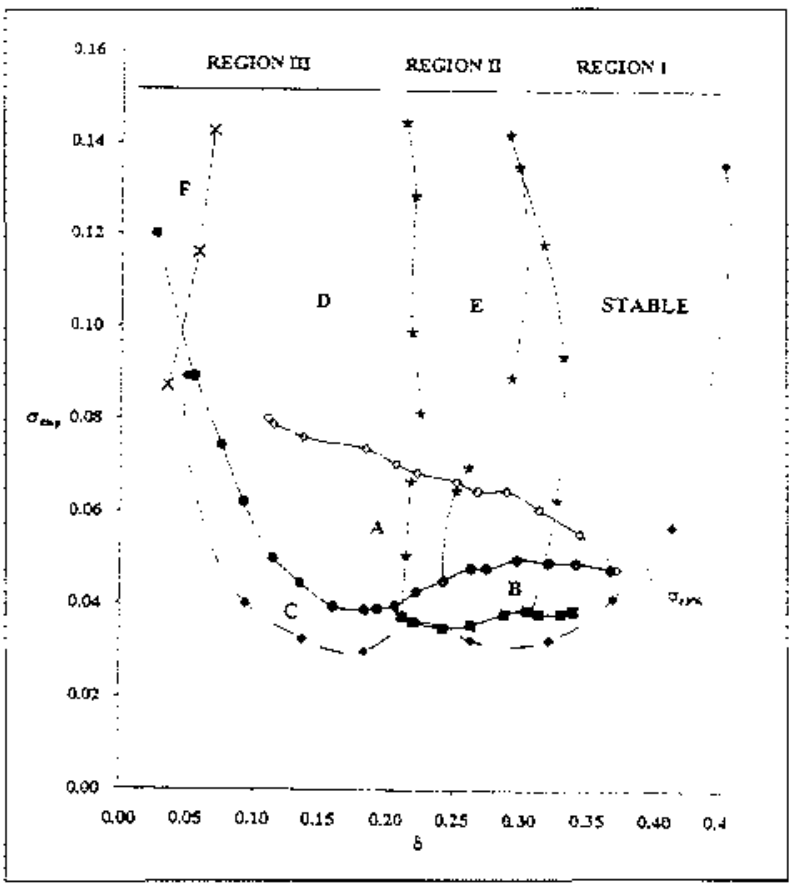

4. Domaines d'instabilités inducteur non fretté [1]

la cavitation de recirculation, puis l'apparition de la cavitation d'aubage en tête qui s'étend progressivement en direction tu pied, pis des pulsations de cavitation qui s'atténuent progress:vement el enfin la disparition des pulsations amenant à un ćcoulement assc $z$ stable.

Lorsque le coefficient de dábil dest inléricur à 0,275 , ta diminution de $\sigma_{\mathrm{d} \text { s }}$ provoque successivement : l'apparition de trois petits cônes de recirculation qui remontent vers l'amont puis la diminution c̀e l'éterdue de cette recirculation avec une augmentation de la fréquence des pulsations. L'écoulement stable óservé pour des débits plus êlevés ne peut être atteint dans ce cas (les periomances de la pompe à vide utilisée ne permetten: pas C'obtenir čc très faibles NPSH).

Quel que soil le débit, l'apparition? ċes pulsations de cavilation est observéc pour des valeuts de $\sigma_{\text {disy }}$ plus faibles que dans le cas de l'inducteur non fretlé.

La présence de la frette empêchant de visualiser l'intérieur de la roue, it est impossible de décécr le moment où la poche de cavitation attein le col du canal interaubes. De même les iấchers de poches visualisés dans le cas non lrelté à l'intérieur du canal interaubes ne sont plus visibles.

\section{ELUCTUATIONS DE PRESSION}

Nous avons mesuré en 123 points répartis dans le plan $\mathrm{NPSH}_{\text {tux }} / \mathrm{Q}$ l'amplitude crête à crête des signaux de pression relevés à $2 \mathrm{~m}$ cn amont de l'inducteur et à $1 \mathrm{~m} \mathrm{cn}$ aval de la roue centrifuge.

\subsection{A l'amont de la machine}

Les courbes isoamplitudes des fuctuations de pression a $2 \mathrm{~m}$ cn amont de l'inducteur présentées sur la figure 5 mettent en ćvidence les mêmes domaines que ceux décelés lors des visualisations. Unc analyse spectrale a ćté réaliséc à partir de la transformée đe Fouricr rapide du signal de pression à $2 \mathrm{~m}$ cn amo:1t de l'inducieur: Les spectres des différents signaux comportent 


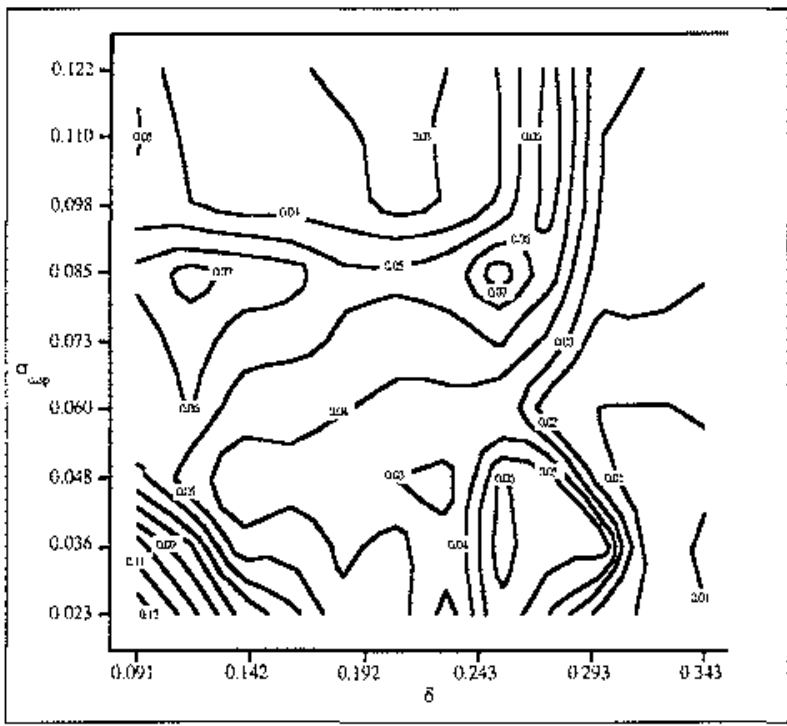

5. Courbes isoamplitudes des fluctuations de pression à $2 \mathrm{~m}$ en amont de l'inducteur fretté.

de manière génćrale trois pics notés $\mathrm{F} 1, \mathrm{~F} 2, \mathrm{~F} 3(0 \mathrm{~Hz} \leq \mathrm{F} 1 \leq 20$ $\mathrm{Hz}, 20 \mathrm{~Hz} \leq \mathrm{F} 2 \leq 80 \mathrm{~Hz}, 60 \mathrm{~Hz} \leq \mathrm{F} 3 \leq 150 \mathrm{~Hz}$ ) d'amplitudes plus ou moins importantes. Les figures $7 \mathrm{a}, 7 \mathrm{~b}$ ct $7 \mathrm{c}$ montrent les amplitudes respectives des pics F!, F2 et F3 dans le plan $\mathrm{NPSH}_{\text {iis }} / \mathrm{Q}$.

Le fonctionnement stable de la région I est confirmé par de Irès faibles fluctuations de pression.

Le domaine B dans lequel des pulsations de cavitation sont observées à la traversée de ia roue de gavage, correspond à des Huctuations de faibles amplitudes, de basses et moyennes fréquences Fl et li2. Il est décalé vers des plus faibles : $\mathrm{NPSH}_{\text {ditrp }}$ dans le cas de l'inducleur fretté

Le domaine C, lieu de manifestation de fortes pulsations de cavitation avec recirculation, présente des fluctuations de pres. sion d’amplitudes très importantes el de basses fréquences. La limite supérieure du domaine $C$ est décaléc vers de plus faibles NPSH ${ }_{\text {dur }}$ dants le cas fretié.

Le domainc $\mathrm{D}$ correspond à des amplitudes de fluctuations relativement faibles gui von ch augmentant lorsoue l'on se rapproche de la zone $\mathrm{F}$ de formation de la torche. Le pic $\mathrm{F}$ l de basscs fréquences est prépondérant dans cc domaine. La limitc droite du domaine est décalée vers des débits plus élevés dans le cas de l'inducteur fretté.

Le domaine $\mathrm{E}$ dans lequel la recirculation apparaît sous la forme d'un tore est le siège de fluctuations de fortes amplitudes. Le pic de hautes fréquences $\mathrm{F} 3$ est très présent dans cc domajne. Ce domaine s'étend sur une plage de débit plus restreinte dans le cas de l'inducteur fretté.

Dans le domaine F, caractérisé par la présence de la torche, les amplitudes des fluctuations sont relativement élevées pour de forts $\mathrm{NPSH}_{\text {ditze }}$ el de plus en plus importantes lorsque $\mathrm{NPSH}_{\text {cis: }}$ diminuc. Le pic FI de basses fréquences est te seul obseryable dans ce domaine.

\section{$4.2 \mathrm{Al}$ l'aval de la machine}

Les amplitudes des fluctuations de pression au refoulement de la pompe présentécs figure 8 sont relativement faibles sur la majeure partic du plan NPSH $\mathrm{Nus}_{\mathrm{g}} \mathrm{Q}$, c'est-à-dire que les fluctuations de la pression amont ne sc propagent pas à travers la roue

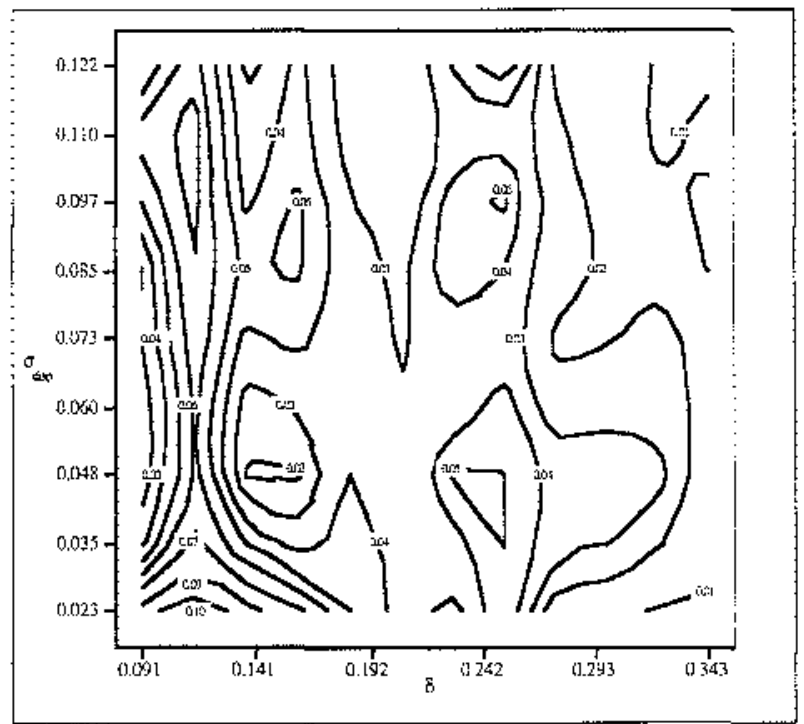

6. Courbes isoamplitudes des fluctuations de pression à $2 \mathrm{~m}$ en amont de l'inducteur non 'retté [1].

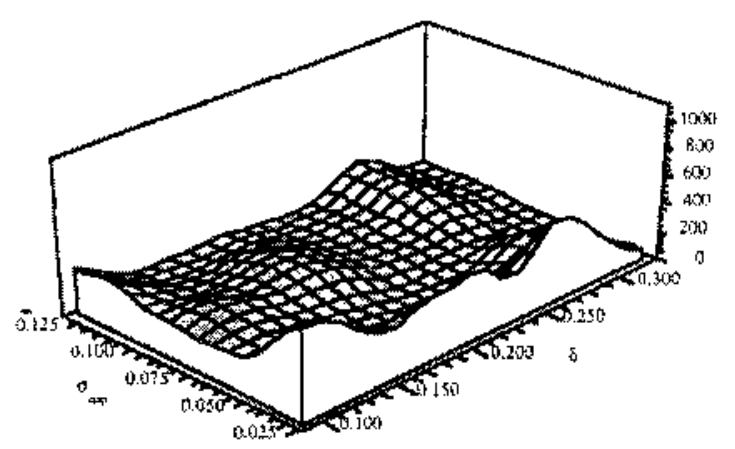

7a. Amplitudes du pic $F_{\text {, }}$

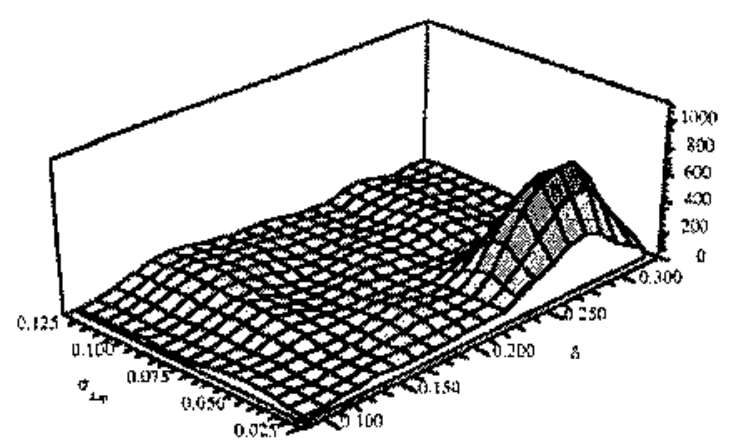

7b. Amplitudes du pic $\mathrm{F}_{2}$

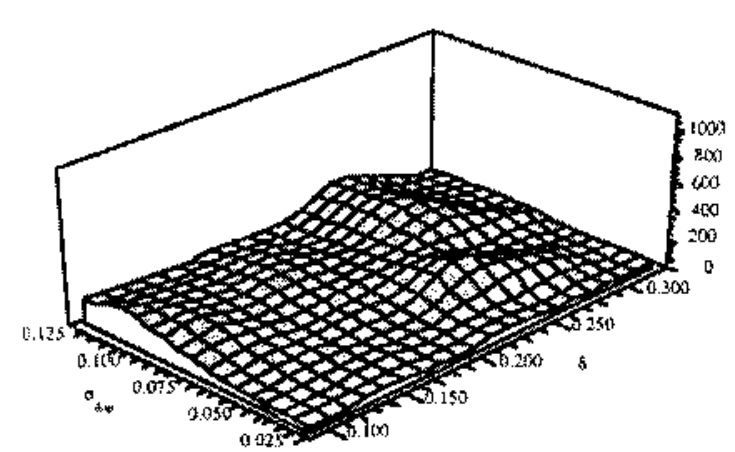

7c. Amplitudes du pic $F_{x}$ 


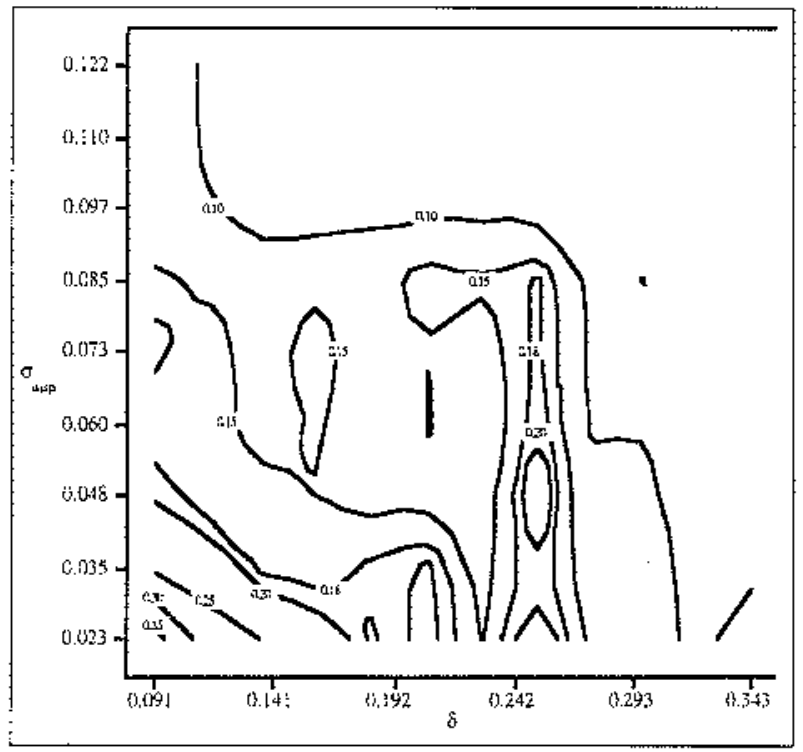

8. Courbes isoamplitudes des fluctuations de pression à $1 \mathrm{~m}$ en aval de la roue centrifuge.

Cas fretté.

centrifuge.

On note cependant de fortes fluctuations pour les faibles débíts cl bas $\mathrm{NPSH}_{\text {disp }}$. Pour cette piage de fonctionnement les fluctuations de très fortes amplitudes visibles à l'amont traversent la rouc. Lne élude des signaux confirme que les pulsations amont et aval sont en phase.

Les amplitudes des fluctuations de pression à $2 \mathrm{~m}$ en amont de t'inducleur et à $1 \mathrm{~m}$ en aval ce la roue centrilıge mesurées dans le cas fretté sont légèrement plus importantes que cans le cas non freté. La présence de la frette n'a pas aténué les instabitités de lonctionnement à débit partiel de :'inducteur. Il se peul que la cavitation marginale ne soit pas aussi néfaste que l'on pouvajt le penser el que, au contraire, celle-ci, en désorganisant les autres formes de cavitation, diminue les Đuctuations de pression associécs.

\section{$V \square$ CONCLUSION}

Les instabilités de fonctionnement de l'inducteur frelté piacé cn alimentation de la pompe centrifuge ont été étudiés. Les campagnes de visualisation et de mestres pariétales instationnaires dans les conduites amont et aval ont permis de déceler dans le plan $\mathrm{NPSH}_{\text {dise }} / \mathrm{Q}$ des domaines de fortes instajilites de fonclionnement de l'inducteur fretté.

Le domaine B dans lequel des pulsations de cavitation sont observées 2 la traversce ce la roue de gavage, correspond à des fluctuations de faibles amplitudes, de basses el moyennes fréquences. Il est décalé vers des țlus faibles $\mathrm{NPSH}_{\text {str }}$ dans le cas de l'inducteur fretcé.

Le domaine $C$, lieu de manifestation de fortes pulsations de cavitation avec recirculation, présente des fluchaations de pression d'amplitudes très imporiantes et de basses fréquences. La limite supéreure de ce domaine cst décalćc vers des plus faibles NPSH $_{\text {ditp }}$ dans le cas fretié.

Le domaine D correspond à des amplitudes de thuctuations eclativement faibles cui vont en augmentan: lor'sue l'on se raj-



9. Courbes isoamplitudes des thuctuations de pression à $1 \mathrm{~m}$ en aval de la roue centrifuge.

Cas non tretréte [1]

proche de la zone de formation de la lorche. Les basses fréquences sont prépondérantes dans ce domaine. La limite droite du domaine cst décaléc vers des débits plus ćlevés dans le cas de l'inducteur fretté.

Le domaine $E$ dans 'eque? Ia recirculation apparaît sous la forme d'un tore montre des lluctuations de fortes amplitudes. Les hautes fréouences sont très présentes dans ce domaine qui s'êtend sur une plage de débil plus restreinte dans le cas de l'inducteur fretté.

Dans le domaine F, caractérisć par la présence de la torche, les fluciuations d'amplitudes sont relativement élevées pour de forts $\mathrm{NPSH}_{d \mathrm{~s} p}$ et de plus en plus importantes lorsque $\mathrm{NPSH}_{\text {tros }}$ diminue. Dans ce domaine seules les basses fréquences sont présentes.

Dans tous jes domaines, les fluctuations de pression dans le cas fretté sont pratiquement identiques à celles du cas non fretté. Ces fluctuations étant nélastes pour le circuit c: la machine, leur alténuation esi envisagéc par un traitement de carter acivellement en cours d'essais.

\section{REMERCIEMENTS}

Cetle étude a été réaliséc cn collaboration avec EDF - DER (Electricité De France - Direction des Eudes et Recherches) ct GEC ALSTHOM - BERGERON.

\section{RÉFÉRENCES}

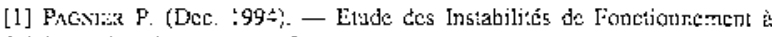
Debi: Partic), de kouts de Gavage (Induceters' er. Alimentation de Pompes Cer:trifiges. 1Y:èse de Doclore: de l'IN'SA de Lyou, 203 p.

[2] $\Lambda \cos T A$ A.J. (1958), ..- An experimental Study of Caviating Inducers Preceedings of Second Sympos:am on Navad Hydrodynamiss, Wastirgton D).C., Aing. 25-29, pp 553-557.

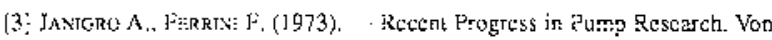
Karman lnstitue lecture scric 61.

14: LAKSIRTKARAYANA 13, (Dec. 1972), ..... Visuatization Study of IFow ir Axia' F:ow Inducer. ASME Journal of Basic Enginecting: pp 777-787.

[5] YFo:D:ar: S. (Miny 1986), - Cerain E.fec:s of Recircliajor on Cavitation

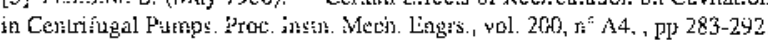

